\section{Good vibrations in cancer}

\section{By Kai-Jye Lou, Staff Writer}

Cancer biomarkers are often present at very low concentrations in the blood, which makes it difficult to separate a positive signal from background noise and can lead to inaccurate or inconclusive blood test results. In addition, a blood test for a cancer biomarker won't give information on the specific location of a tumor.

Looking to address both issues, researchers at the Stanford University School of Medicine have shown that localized application of ultrasound can be used to amplify the release of biomarkers into the blood. ${ }^{1}$ They have used ultrasound to increase the release of an established cancer biomarker-carcinoembryonic antigen (CEA) - in mice.

Ultrasound energy makes cell membranes more permeable, and multiple research groups have exploited this phenomenon to increase the delivery of macromolecules into cells. The Stanford team, however, took the concept and reversed it.

"Because ultrasound could facilitate the delivery of macromolecules into the cell, we thought that maybe it could also be used to facilitate the release of macromolecules from the cell," said Sanjiv Gambhir, a professor in the Department of Radiology and the Department of Bioengineering and director of the molecular imaging program and Canary Center for Cancer Early Detection at Stanford. "The underlying hypothesis here is can we subject tumors to an external energy source like ultrasound to change the amount of biomarkers released into the blood, rather than having to wait months or years for the tumor to grow and increase the amount of biomarkers that it releases."

Ultrasound is routinely used to image soft tissues, but when applied at higher intensities its energy can stress and kill cells. The Stanford group focused on identifying ultrasound protocols that could amplify biomarker release into the blood without inducing significant cell death. "We don't want to deliver too much energy so that we kill the exposed cells, nor do we want to deliver too little and not trigger biomarker release," Gambhir told SciBX. "It was a lot of trial and error to find ultrasound protocols that worked."

The researchers were able to identify ultrasound protocols that work in vitro and in vivo. In a human colon cancer cell line, exposure to ultrasound caused a threefold to fourfold increase in CEA levels released into the media compared with no ultrasound ( $p=0.036$ ).

The team also showed that ultrasound can help reveal a tumor's location. In mice, application of ultrasound to a tumor-containing region significantly increased serum Cea, but application to a tumorfree region did not $(p<0.031)$.

Data were published in the Proceedings of the National Academy of Sciences.

\section{Going broader}

The Stanford group thinks the approach could improve early disease detection of certain cancers that are both accessible by ultrasound and associated with validated serum biomarker assays.

"Ultrasound could radically change our approach to biomarker detection because it provides physicians with the ability to stress the tumor and trigger biomarker release," said Gambhir, who is the co-corresponding author on the paper. "Blood biomarker levels can vary a lot from patient to patient and even at different times within the same patient. The nice thing about the ultrasound technique is that patients become their own control," he said.

"I think this approach may provide a way to improve the ability for tests to detect cancer biomarkers," said David Ransohoff, director of the clinical research curriculum and a professor of medicine at The University of North Carolina at Chapel Hill School of Medicine. "By shaking the biomarkers loose from cells and releasing them into the bloodstream, these markers may become easier to detect."

Individuals at risk for developing tumors in highly susceptible areas like the breasts and pelvic regions could be the most likely candidates for early detection tests that use the ultrasound approach. The at-risk group includes individuals with family histories of cancer or known genetic predispositions like mutations in the breast cancer 1 early onset (BRCA1) and BRCA2 genes, or previously treated patients.

However, because ultrasound is reflected at air-to-tissue interfaces, it may not be feasible to use the approach in tumor-prone regions like the lung and bowel.

To screen for possible tumors and their location, physicians could obtain a blood sample pre-ultrasound and use that as the baseline for a sample taken post-ultrasound. Because ultrasound exposure is localized to specific regions of the body, increased biomarker levels in a blood sample taken post-ultrasound also would provide spatial information on the origin of biomarker release.

"One could use ultrasound to localize disease by sonicating a region of suspicion in patients at risk for cancer," said Michael Kolios, an associate professor in the Department of Physics at Ryerson University and the Canada Research Chair in Biomedical Applications of Ultrasound. "I think this could have a big impact for studying different tumors and in looking for new biomarkers in preclinical models."

However, Kolios noted that the mice used in the Stanford study had large tumors, which may not be representative of an early detection scenario. 
"Usually, biomarker screens are used to detect something early on. However, the tumors used in the study are pretty large relative to the size of animals," he said. "The researchers will need to evaluate the ultrasound approach in enhancing the detection of de novo tumors."

Samir Hanash, program head of molecular diagnostics at the Fred Hutchinson Cancer Research Center, wants to see whether the ultrasound approach works with other cancer biomarkers. "All the current data is based on one protein, so it is hard to know whether this protein is an exception or a general trend," he said. "The question that still needs to be answered is how applicable is the approach going to be for any kind of cancer."

Indeed, it may be difficult to measure the concentration of biomarkers that have short plasma half-lives. Also, humans have a much larger volume of blood than mice, so potential dilution of a biomarker's signal would need to be addressed in future studies.

\section{Sights and sounds}

Gambhir said the Stanford group is looking to answer those questions.

The team is evaluating the use of ultrasound-mediated biomarker amplification in additional tumor types and biomarkers in animal models. He added that the group also will need to identify ultrasound protocols that work in different parts of the body.

Within a year, Gambhir hopes to begin evaluating the technology in the clinic.

"For translation to the clinic, we want to start by looking at patients with tumors and measure biomarker levels in blood before and after the application of ultrasound," he said. "Then we will evaluate its use in high-risk patients. We could apply ultrasound to a suspected region in high-risk patients and see if there is a corresponding increase in blood biomarker levels."

Gambhir said the group initially will evaluate ultrasound-mediated biomarker release from tumors in the breast and pelvis, which are tumor-prone regions that are accessible with ultrasound.

Stanford has a pending patent covering methods to spatially localize and amplify biomarker levels, in blood or other biological fluids, by applying external energy sources like ultrasound. The work is available for licensing from the Stanford University Office of Technology Licensing.

Lou, K.-J. SciBX 2(41); doi:10.1038/scibx.2009.1524

Published online Oct. 22, 2009

\section{REFERENCES}

1. D'Souza, A.L. et al. Proc. Natl. Acad. Sci. USA; published online Sept. 21, 2009; doi:10.1073/pnas.0903437106

Contact: Gary M. Glazer, Stanford University School of Medicine, Stanford, Calif.

e-mail: glazer@stanford.edu

Contact: Sanjiv S. Gambhir, same affiliation as above e-mail: sgambhir@stanford.edu

\section{COMPANIES AND INSTITUTIONS MENTIONED}

Fred Hutchinson Cancer Research Center, Seattle, Wash. Ryerson University, Toronto, Ontario, Canada

Stanford University School of Medicine, Stanford, Calif. The University of North Carolina at Chapel Hill School of Medicine, Chapel Hill, N.C. 